\title{
A new approach to study energy-dependent arrival delays on photons from astrophysical sources
}

\author{
Manel Martínez * and Manel Errando \\ Institut de Física d'Altes Energies, Edifici Cn Universitat Autonòma de \\ Barcelona, 08193 Bellaterra, Spain
}

\begin{abstract}
Correlations between the arrival time and the energy of photons emitted in outbursts of astrophysical objects are predicted in quantum and classical gravity scenarios and can appear as well as a result of complex acceleration mechanisms responsible for the photon emission at the source. This paper presents a robust method to study such correlations that overcomes some limitations encountered in previous analysis, and is based on a Likelihood function built from the physical picture assumed for the emission, propagation and detection of the photons. The results of the application of this method to a flare of Markarian 501 observed by the MAGIC telescope are presented. The method is also applied to a simulated dataset based on the flare of PKS 2155-304 recorded by the H.E.S.S. observatory to proof its applicability to complex photon arrival time distributions.
\end{abstract}

Key words: Lorentz invariance, quantum gravity, Very High Energy gamma-rays, Active Galactic Nuclei, MAGIC, H.E.S.S.

\section{Introduction}

Observations of the electromagnetic radiation from astrophysical sources exhibiting fast flux variations - mainly active galactic nuclei (AGNs) and gammaray bursts (GRBs) and pulsars- make possible the study of correlations between the energy and the arrival time of individual photons. Two different effects can explain the appearance of such correlations: either they are sourceintrinsic or have originated during the propagation of the radiation from the

\footnotetext{
* Corresponding author. Tel. +34 935811309, Fax +34 935811938.
}

Email address: martinez@ifae.es (Manel Martínez). 
source to the Earth. On the one hand, the emission mechanisms taking place at the source can cause delays between photons of different energies to appear. In the case of pulsars, a difference in the emission location for different energies can cause the shape and peak time of the pulses to change with energy [12]. In AGNs the delays can be caused, for instance, by the acceleration of the electrons responsible for the emission [3] 4 . On the other hand, if the emission is assumed to be simultaneous at the source for all the photons in a certain energy range, an energy-dependent propagation effect could also explain the appearance of these correlations. Quantum Gravity (QG) [5,6,7,8,9] and classical gravitation -through the existence of wormholes [10]- are two frameworks where this kind of propagation effects may show up.

It is widely speculated that space-time is a dynamical medium, subject to quantum-gravitational effects that cause space-time to fluctuate on the Planck distance scales. Model realizations of QG predict that quantum fluctuations in the space-time metric make it appear 'foamy' on very short distance scales (see [11,12,13,14] for reviews). The propagation of photons through this fluctuating space-time might induce an energy dependence of the speed of light, resulting in an observable difference on the propagation time for photons of different energies. The anomaly induced by QG will always be a small perturbation of the assumed light speed $c$, and can therefore be treated as a correction of the form $\Delta c / c=-E / M_{Q G 1}$ or $-E^{2} / M_{Q G 2}^{2}$ where $E$ is the photon energy, $M_{Q G 1}$ and $M_{Q G 2}$ are respectively the effective $Q G$ scales in the linear and the quadratic term, and the minus sign indicates that most of the models expect this effect to be subluminal.

The possibility to observe QG efects in the propagation of photons from astrophysical sources was proposed by Amelino-Camelia et al. [15]. If two photons of different energy are emitted simultaneously from an astrophysical object, the expected delay between their arrival times when they are detected increases with the distance to the source and with the energy of the photons. Therefore, the maximum sensitivity to energy dependent propagation effects is expected from observations of very fast flux variations coming from sources at large distances that emit photons up to very high energies.

The first candidates considered for such observations were gamma-ray bursts (GRB) [15], but the first experimental result on possible energy-dependent speed of light came from the measurement of a flare of the active galactic nucleus (AGN) Mkn 421 at TeV energies by the Whipple gamma-ray telescope [16], claiming a lower limit to the energy scale of quantum gravity of $M_{Q G 1}>6 \times 10^{16} \mathrm{GeV}$. Other bounds have been obtained by studying the emission of pulsars [17], and a combined analysis of many GRBs yielded to a robust lower limit of $M_{Q G 1}>0.9 \times 10^{16} \mathrm{GeV}$ [18].

The highest sensitivity of the new generation of ground-based gamma-ray 
telescopes was expected to have a clear impact in these studies [19] and, indeed, has provided new observations of fast flares of AGNs at TeV energies with richer photon statistics than the previous generation of instruments could [20,21], enhancing the sensitivity of these measurements to any energy dependent propagation effect.

This paper presents a new method of analysis that can be applied to any observed set of photons to search for any kind of correlations between their arrival time and energy. This analysis technique is described in section 2 and applied to the Mkn 501 flare observed by the MAGIC telescope in July 2005 [20] in section 3. An application to a simulated set of photons based on the more complex flare structure of the PKS 2155-304 outburst observed by the H.E.S.S. collaboration in July 2006 [21] is discussed in section 4. Finally, the conclusions of this work are summarized in section 5 .

\section{Description of the method}

The pioneering search for QG efects using TeV gamma-ray data by the Whipple Collaboration [16] was based on few tens of gamma-ray events that where both binned in time and energy.

Given the fact that the statistics for sources at the largest distances from us and photons of the highest energies (conditions that maximize the expected photon delay) is usually scarce, in order to make an optimal use of the information of the arrival time and estimated energy of each recorded photon the analysis method used should be unbinned.

Some analysis methods, such as the Modified Cross Correlation Function (MCCF) [22,23] or the Continuous Wavelet Transform (CWT) [24] have been widely used for similar analysis [18,25,26,27,28]. These methods have been applied using the information of the arrival time for each individual photon without binning, but binning the energy in bands in order to determine the time lag as a function of the energy and therefore did not make optimal use of the photon information.

In addition, as mentioned before, measurements at the highest photon energies maximize the expected delays between photons, but all the sources detected at the GeV-TeV energy range show steeply falling energy spectra, reducing the photon statistics as the energy increases. Some of the above analysis methods, as for instance the wavelet approach, 25] simply cannot deal properly with the low photon statistics obtained in most of the very high energy gamma-ray measurements. 
Completely unbinned approaches to search for correlations in similar data sets are nevertheless possible. For instance the Energy Cost Function (ECF) method described in [29] has been successfully used to measure a tiny time lag in the same data set which will be used here as an example. Nevertheless, that method requires the identification of well-isolated flares and cannot be applied directly to complex lightcurves where several overlapping flares, or simply other kind of features such as edges of non-contained flares, appear in the lightcurves.

This work describes a completely unbinned method which overcomes the above limitations and can be applied in a general manner to any data set, regardless on the photon statistics and the lightcurve shape and structure. Moreover, it makes use of an optimal estimator which, if properly applied, would use all the information contained in the data and therefore provide with the most accurate estimate of the correlation between energy and arrival time. This estimator is the Likelihood Function built from the combined probability of having observed a set of photons with individual energy $E_{i}$ arriving at time $t_{i}$, and has to be constructed from a physical description of the assumed processes involved in the emission, propagation and detection of the photons.

Any hypotheses about the physics mechanisms that finally produce the measured set of photons can be accommodated in this analysis provided a reasonably simple mathematical description is at hand. A possible general physics picture of the process is the following:

- Photons were produced at the source following an intrinsic lightcurve ${ }^{1}$ $F_{s}\left(t_{s}\right)$ where $t_{s}$ is the time at the source and an intrinsic energy spectrum $\Gamma\left(E_{s}\right)$ where $E_{s}$ is the energy of the photons at the source. It might be that already at the source the lighcurve depends on energy or the energy spectrum depends on time. If models describing these dependencies are at hand for the observed astrophysical source, they can be readily incorporated in the approach described here.

- These photons propagate in space and there, if there is an energy-dependent refraction index related to some effective energy scale parameter $M_{Q G n}$, a delay $D\left(E_{s}, M_{Q G n}, z\right)$ should affect their propagation time.

- These photons reach the Earth and are recorded by satellite or groundbased instruments as photons with measured energy $E$ and arrival time $t$. These detectors have typically an extremely good time resolution compared with the flare time scales but a limited energy resolution $G\left(E-E_{s}, \sigma_{E}\left(E_{s}\right)\right)$ which may be any complex function of the observed energy.

1 The term "lightcurve" is widely used in astrophysics to denote the photon emission time distribution function. 


\subsection{Probability Density Function}

A mathematical expression casting the probability density function (p.d.f.) describing the physics picture given above is the following:

$$
\frac{d P}{d E d t}=N \int_{0}^{\infty} \Gamma\left(E_{s}\right) C\left(E_{s}, t\right) G\left(E-E_{s}, \sigma_{E}\left(E_{s}\right)\right) F_{s}\left(t-D\left(E_{s}, M_{Q G n}, z\right)\right) d E_{s}
$$

where

- $P$ is the probability density function (p.d.f.) for a photon to have observed energy $E$ and arrival time $t$.

- $\Gamma\left(E_{s}\right)$ is the photon energy distribution at the source. For instance, for Very High Energy gamma rays, in most cases the energy spectrum is well described in first approximation by a pure power law $\Gamma\left(E_{s}\right)=\Gamma_{0} \cdot\left(E_{s} / E_{0}\right)^{-\alpha}$ where $\alpha$ is the differential spectral index. Nevertheless, more complex functions such as broken or curved power laws, spectral index for the photons coming from the flare activity different from the spectral index for the photons coming from the steady activity, or synchrotron or inverse Compton spectra can be readily used. The spectrum can be obtained from a fit to the whole photon dataset assuming that it does not change during the flare. Otherwise, if the photon distribution is observed to change with time it can be fit to the data assuming a specific mechanism for correlation at the source.

- $C\left(E_{s}, t\right)$ accounts for changes in the effective area of the detector during the observation of the flare. In most cases this factor will be constant and can be neglected. It has to be included, for example, if the data comes from ground-based telescopes with changing observation conditions (significant changes in the zenith angle of the pointing, atmospheric conditions, etc.) or from satellites observing in survey mode.

- $G\left(E-E_{s}, \sigma_{E}\left(E_{s}\right)\right)$ is the gamma energy smearing produced by the instrument. In first approximation it could be a gaussian distribution with width $\sigma_{E}\left(E_{s}\right)$ although more complex functions can also be used. This function has to be obtained from the study of the energy response of the detector.

- $D\left(E_{s}, M_{Q G n}, z\right)$ is the eventual propagation delay as a function of the photon energy $E_{s}$, an effective energy scale $M_{Q G n}$ and the source redshift $z$. This function may accommodate linear, quadratic or any arbitrary realization of any possible propagation delay mechanisms. A general implementation of this function in terms of an effective expansion in the case of Quantum Gravity scenarios is discussed below.

- $t_{s}=t-D\left(E_{s}, M_{Q G n}, z\right)$ is the actual photon production time at the source obtained from the measured arrival time corrected by the delay generated 
by non-trivial propagation effects.

- $F_{s}\left(t_{s}\right)$ is the emission time distribution of the photons at the source. For most astrophysical sources there are no compelling models which can be used to predict and/or fit the flaring lightcurves. Moreover, since the probability $P$ depends only on the relative delay of the photons for different energies, the best estimator for the time structure at the source can be taken to be the one of the observed gamma emission time distribution for the lowest energy gamma rays. In the specific case of Very High Energy gamma rays, this estimator is a very good choice because, since the energy spectra are typically steep power laws, most of the recorded gammas are collected at the lowest energies, and therefore the gamma statistics is then sufficient to infer the lightcurve as explained above.

- $N$ is the p.d.f. normalization. This normalization is extremely important for the proper use of the probability in a likelihood function. To have an unbiased estimation of the fitting parameters it has to be computed in such a way that the p.d.f. integral is equal to 1 no matter the values of the free parameters in the p.d.f.

If the propagation delays are inspired in Quantum Gravity models, in a power $n$ realization of Quantum gravity effects ( $n=1$ linear and $n=2$ quadratic) for $z<<1$ we have that the propagation delay of a photon of energy $E_{s}$ with respect to the arrival time of a photon of energy $E_{s, 0}$ is:

$$
D\left(E_{s}, M_{Q G n}, z\right)=\frac{E_{s}^{n}-E_{s, 0}^{n}}{M_{Q G n}^{n}} \frac{z}{H_{0}}
$$

where $z$ is the source redshift and $H_{0}$ is the local Hubble constant (in inverse seconds). For sources at larger distances the redshift in the photon energies and the expansion effect in the photon path have to be taken into account, and then the correct expressions are:

$$
D\left(E_{s}, E_{Q G}, z\right)=\frac{1}{H_{0}} \frac{E_{s}-E_{s, 0}}{M_{Q G 1}} \int_{0}^{z} \frac{(1+z) d z}{h(z)}
$$

for the linear case and

$$
D\left(E_{s}, E_{Q G}, z\right)=\frac{1}{H_{0}} \frac{E_{s}^{2}-E_{s, 0}^{2}}{M_{Q G 2}^{2}} \int_{0}^{z} \frac{(1+z)^{2} d z}{h(z)}
$$

for the quadratic case where

$$
h(z)=\sqrt{\Omega_{\Lambda}+\Omega_{M}(1+z)^{3}}
$$


being $\Omega_{\Lambda}$ and $\Omega_{M}$ the standard cosmological parameters.

\subsection{Likelihood Function and fitting procedure}

With the above described p.d.f. the likelihood function for the observation of all the $N_{\gamma}$ photons recorded during the flare can be build as

$$
L=\prod_{1}^{N_{\gamma}} \frac{d P}{d E_{i} d t_{i}}
$$

In the above expression, several free parameters may appear. One of them is obviously the effective scale $M_{Q G n}$ at which propagation delays appear. Other parameters may try to fit the intrinsic lightcurve shape to some analytic or semi-analytic parametrization or the intrinsic energy spectrum. The likelihood function can be fitted to the data using a numerical procedure to find the set of free parameters that minimize the function $-2 \log (L)$.

From the conceptual point of view, when the $M_{Q G n}$ parameter is changed, the effect is basically sliding in time the lightcurve profile as a function of energy to fit the possible correlation between energy and arrival time in the data. If there is no correlation between energy and time, or the correlation is not significative that would correspond to $M_{Q G n} \rightarrow \pm \infty$ and therefore $M_{Q G n}$ is not a good fitting parameter for the expected small correlations, because the likelihood function would be highly non-parabolic. Instead, a well-behaved fitting parameter is $\hat{M}_{P} / M_{Q G n}$, where $\hat{M}_{P}=2.4 \times 10^{18} \mathrm{GeV}$ is the reduced Planck mass, which can have positive or negative or zero values and will likely show a nice parabolic behavior. Moreover, in most QG scenarios its order of magnitude is expected to be around 1 which makes numerical calculations easier.

In the physics picture assumed, when sliding in time the lightcurve all gammas are expected to lead to the same "intrinsic" lightcurve, therefore all gammas, regardless on their energy, contribute in the fitting procedure to either determine or test the assumed lightcurve shape even if just the lower energy ones were initially used to estimate the shape of the lightcurve.

If the fit is well behaved, the fit results for all free parameters shall show a parabolic behavior of the log-likelihood function around the "best fit minimum". Around that minimum, an increase of $\Delta(-2 \log (L))=1$ will then give the single-parameter one- $\sigma$ uncertainties.

Since the Likelihood p.d.f. by construction has dimensions $[E]^{-1}[t]^{-1}$, its value at the minimum does not have a direct interpretation and does not provide a 
simple means to estimate the goodness-of-fit for the assumed function. Therefore additional tests must be performed to quantify the adequacy of the physics picture casted in the Likelihood function to describe the data.

One simple possibility that we have used is the following: once the Likelihood fit is done, the data and the Likelihood function computed at the best fit parameter values are binned in energy and time. Then the $\chi^{2}$ value of the comparison of the resulting bi-dimensional histograms (in energy and time) is computed and compared with the number of degrees of freedom, providing a goodness-of-fit probability. This approach allows also an easy visualization on the adequacy of the fitting function to describe the data. Other possibilities such as integrating the likelihood function would require the use of complex and lengthy multi-dimensional integrations.

\section{Example of application to a Markarian 501 flare}

The blazar Markarian 501, located at $z=0.034$, showed a very high activity in the gamma-ray band in June and July 2006. On July 9 a very strong flare in the $\mathrm{TeV}$ regime was observed by the MAGIC collaboration [20]. The analysis procedure explained in the previous section was applied to this set of photons in reference [29] together with a method based on maximizing the total energy in the most active part of the flare.

In that data sample, the lightcurve was tested to be well described by a simple gaussian flare on top of a baseline constant in time within the observation window (see Fig. 11). It was tested that other mathematical functions fitting also well the data flare shape, such as the halving-doubling time formula used in the original publication [20 or even a simple triangle function, were producing similar final results. Therefore, the time distribution was parameterized as a gaussian flare of width $t_{W}$ and position $t_{0}$ relative to the first gamma arrival time, on top of a flat background time distribution (see figure 1).

The intrinsic spectrum was obtained from a global fit to the flare data and consisted of two power-laws $\Gamma\left(E_{s}\right)=\left(E_{s} / 1 \mathrm{TeV}\right)^{-\alpha}$ with different spectral indices: $\alpha=2.4$ for the gaussian excess component and $\alpha=2.7$ for the continuous baseline [20]. These values were changed within few standard deviations without a significant impact on the final results.

The energy resolution was assumed to be linear with energy $\sigma_{E}=0.22 \times E$ although several other more complex energy dependencies consistent with the MAGIC telescope energy resolution measurements were tested with no significant impact in the results. 


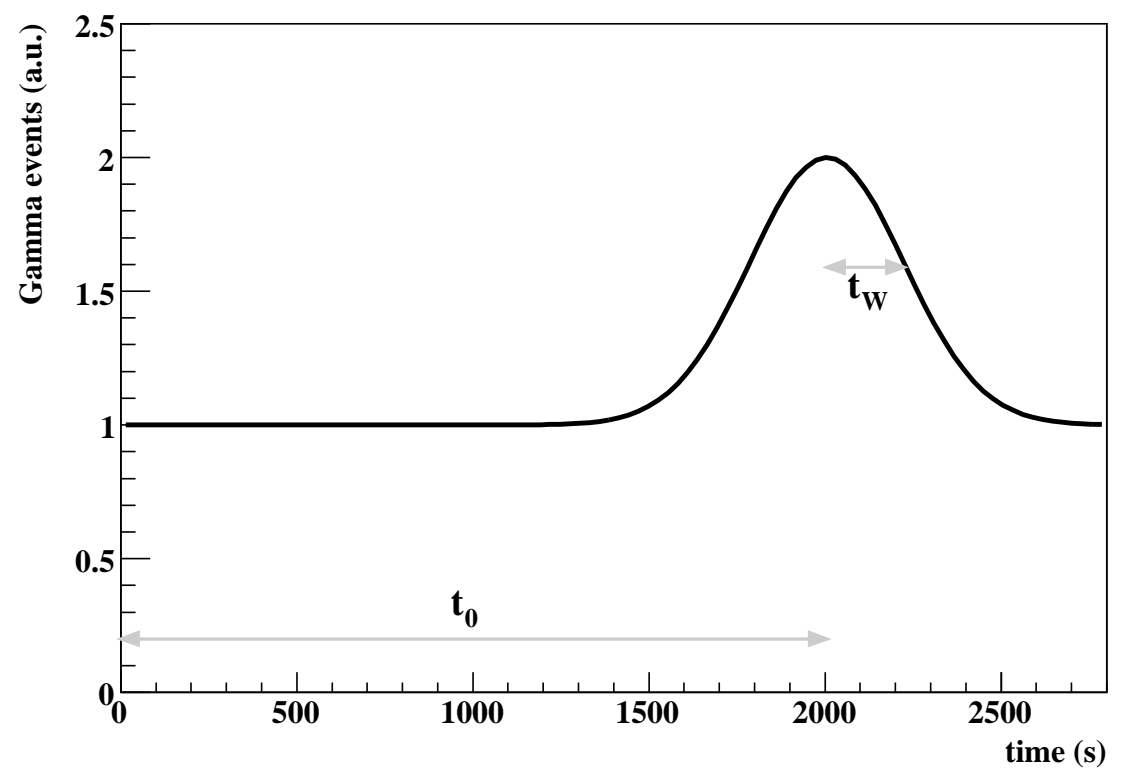

Fig. 1. Parameterized flare shape for the Markarian 501 MAGIC flare data: the data are well described by a Gaussian-shaped flare activity on top of a constant baseline of steady gamma-ray emission (called "baseline" elsewere in this work).

To infer from the data the best estimators for the fitting parameters, the likelihood function was fitted to the data using a standard numerical minimization package to minimize the function $-2 \log (L)$ as a function of four free parameters:

- $\hat{M}_{P} / M_{Q G n}$ the effective energy scale for a linear or a quadratic Quantum Gravity realization.

- $t_{W}$ the gaussian intrinsic flare width.

- $t_{0}$ the maximum intrinsic flare position relative to the first gamma arrival time.

- $x_{B}$ the normalization in area between the flat baseline component and the superimposed gaussian flare.

The fit converged smoothly towards a minimum at $M_{Q G 1}=0.30_{-0.10}^{+0.24} \times 10^{18}$ $\mathrm{GeV}$ for the linear case and $M_{Q G 2}=0.57_{-0.19}^{+0.75} \times 10^{11} \mathrm{GeV}$ for the quadratic one. The uncertainties given above are asymmetric because they are given on $M_{Q G n}$ and not on $\hat{M}_{P} / M_{Q G n}$, the actual fitting parameter, and correspond to the change in the fitting parameter leading to an increase of $\Delta(-2 \log (L))=1$ around the minimum. The shape of the Likelihood function as a function of $\hat{M}_{P} / M_{Q G 1}$ around that minimum is almost parabolic as can be gleaned from Fig. 2 and therefore, the above uncertainty can be considered as enclosing one-sigma single parameter statistical uncertainty. Therefore, the fitted value of $\hat{M}_{P} / M_{Q G 1}$ differs from zero by over two-sigma. 


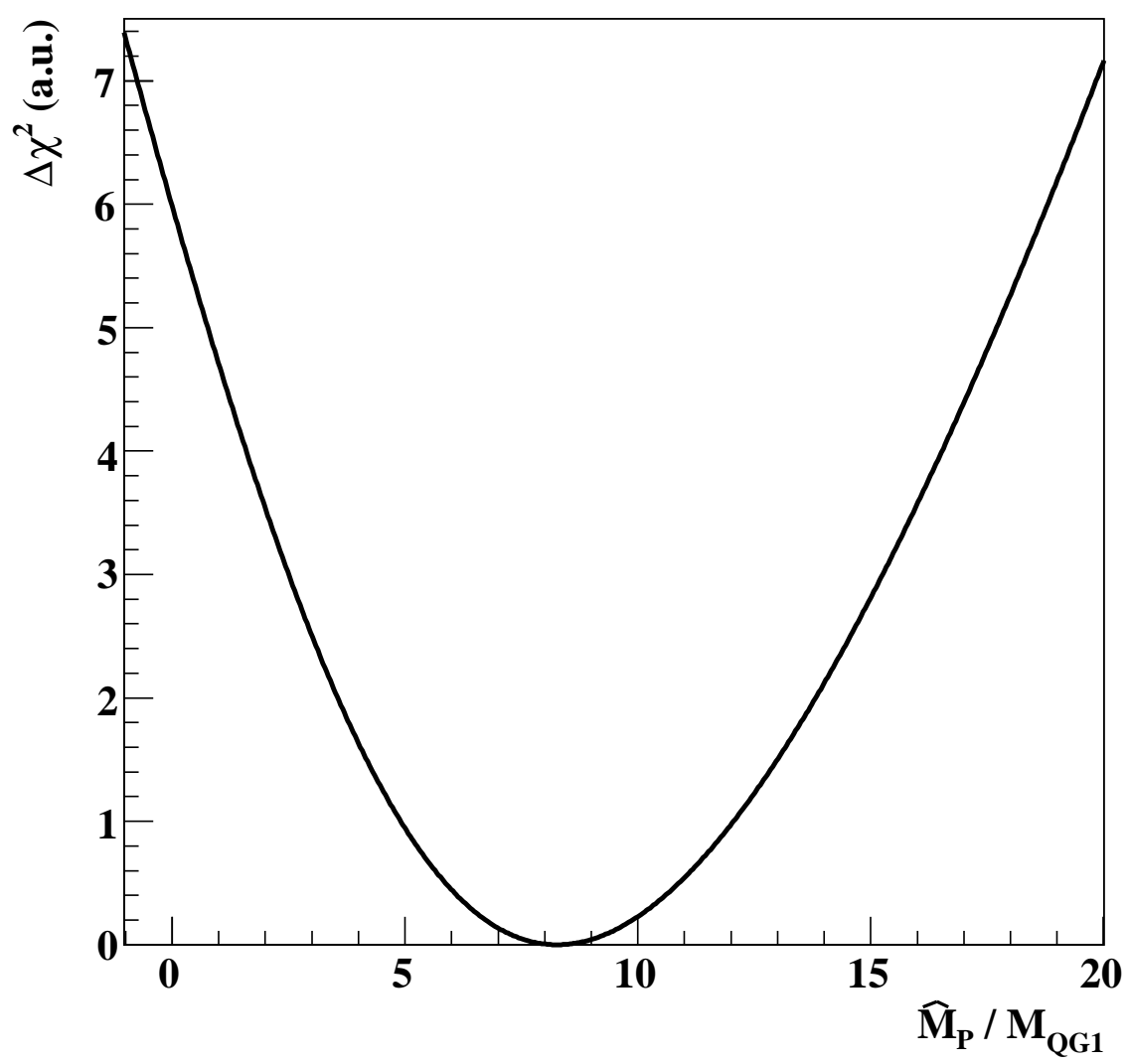

Fig. 2. Behavior of the function $-2 \log (L)$ with respect to the parameter $\hat{M}_{P} / M_{Q G 1}$ around the minimum. The shape is rather parabolic and allows excluding the value $\hat{M}_{P} / M_{Q G 1}=0$ at more than 2 sigma significance $(\Delta(-2 \log (L))=4)$.

The values of the fitting parameters in the linear and quadratic scan are shown in Tab. 1, As discussed in reference [29] this result is perfectly consistent with the one obtained by using the Energy Cost Function method described there. The correlation between the different parameters is always below $50 \%$ except between $M_{Q G n}$ and $t_{0}$, which are correlated at a level of $60 \%$ as the first parameter carries the information about the delay introduced by a QG effect during the propagation of photons and the second indicates where the maximum of the flare is located.

The goal of this work is just the discussion of the new method described here and therefore we shall not discuss here the meaning or the interpretation of the above results. That is properly discussed in reference [29].

Moreover, a series of tests were performed to test the robustness, the stability and the interpretation of the result.

First, the dataset was fitted to the same Likelihood function after scrambling randomly the arrival time of the photons. The fit then produced a best fit 
Table 1

\begin{tabular}{ccc}
\hline \hline & $n=1$ & $n=2$ \\
\hline$\hat{M}_{P} / M_{Q G n}$ & $8.9_{-4.5}^{+5.8}$ & $4.3_{-1.7}^{+2.7} \times 10^{7}$ \\
$t_{W}$ & $219_{-29}^{+28} \mathrm{~s}$ & $(228 \pm 27) \mathrm{s}$ \\
$t_{0}$ & $(2005 \pm 42) \mathrm{s}$ & $(2041 \pm 36) \mathrm{s}$ \\
$x_{B}$ & $0.38 \pm 0.04$ & $0.38 \pm 0.04$ \\
\hline
\end{tabular}

Best fit values of the free parameters in the Likelihood function for the linear and the quadratic scan. These values are obtained when all the parameters of the fit are left free, while the $M_{Q G n}$ values quoted in the text are obtained fixing all the parameters except for $\hat{M}_{P} / M_{Q G n}$ itself.

value compatible with zero energy-time correlation at one sigma.

Second, mock simulated Monte Carlo datasets of the size of the actual data but with input parameters unknown by us were produced ${ }^{2}$ and then fitted blindly by our Likelihood function, recovering the input parameters within the expected uncertainties. The same happened with the Energy Cost Function method as discussed in reference [29].

Third, thousand datasets of the same characteristics of the actual data were produced using a bootstrap method on the actual data. Each of these datasets was fitted to our Likelihood function and the distribution of the best-fit values was studied (see figure 3). As expected from a probabilistic interpretation of the statistical uncertainties obtained in the Likelihood fit to the MAGIC data, the $M_{Q G 1}=0.30_{-0.10}^{+0.24} \times 10^{18} \mathrm{GeV}$ was enclosing about $68 \%$ of the best-fit values and less than $2 \%$ of the best-fit values were below zero.

Therefore, it can be concluded that the Likelihood method explained here was producing in this case a reliable result. Moreover, reasonable modifications on the actual details of the physical process description casted in our Likelihood formula, as already discussed before, change the results in just a fraction of the statistical uncertainty.

Finally, in order to check the goodness-of-fit of our Likelihood formula to the data, both the Likelihood function and the data have been binned following the binning originally used in reference [20], namely: four bins in energy and twelve bins in time (see Fig. 4). The $\chi^{2}$ obtained by comparing the data and fitting function histograms turns out to be in reasonable agreement with the number of degrees of freedom (equal to the number of used bins minus the number of fitting parameters). Therefore, as can be gleaned from Fig. 4, our Likelihood function is indeed a good description of the MAGIC flare data.

2 These datasets were independently produced by Adrian Biland with a toy Monte Carlo simulation implementing his own simple description of the physical process. 


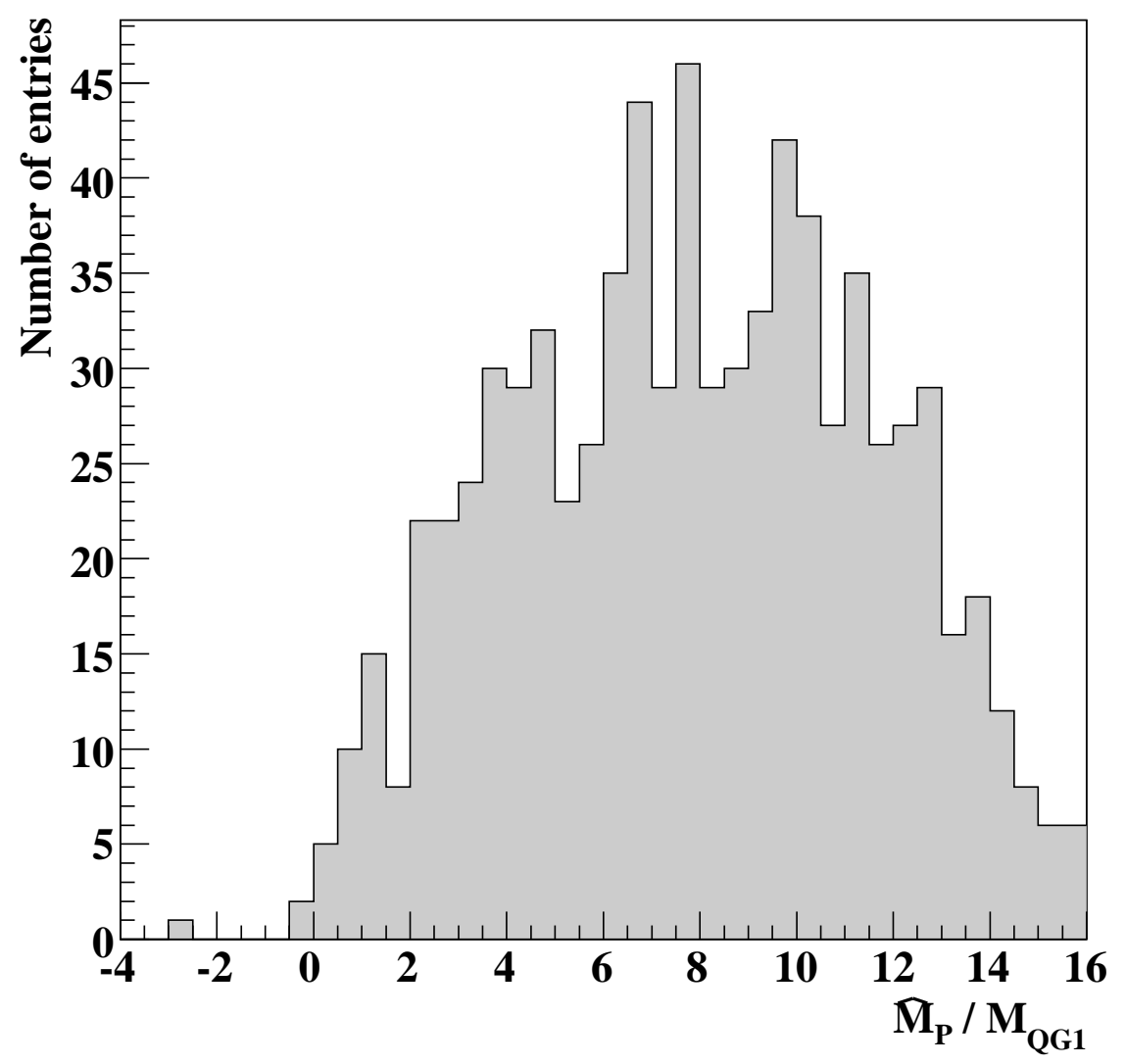

Fig. 3. Distribution for the best fit parameter $\hat{M}_{P} / M_{Q G 1}$ in bootstrap replica of the MAGIC flare data. As can be seen, just few fits produce $\hat{M}_{P} / M_{Q G 1} \leq 0$.

\section{Example of application to simulated photons following a more complex flare structure}

The H.E.S.S. collaboration published in year 2007 the observation of a huge flare of the blazar PKS 2155-304 [21], located at $z=0.116$. The flare happened during the night of July 26th 2006, lasted about 90 minutes and was so intense (over 10 thousand gamma rays collected corresponding to a significance of almost 160 sigma) that a lightcurve with 1-minute time binning was produced.

That lightcurve, shown in Fig. 5, exhibits a rather complex structure in time which, as discussed in reference [21, is well fitted by a set of five overlapping flares described by using "generalized Gaussian" shapes following the formulation suggested by Norris et al. [30], that is:

$I(t)=A \exp \left[-\left(\left|t-t_{\max }\right| / \sigma_{r, d}\right)^{\kappa}\right]$

where $\sigma_{r}$ (rise) is to be used for $t<t_{\max }$ and $\sigma_{d}$ (decay) is to be used for $t \geq t_{\max }$. The actual best fit values for $A, \sigma_{r, d}$ and $\kappa$ for each one of the five 

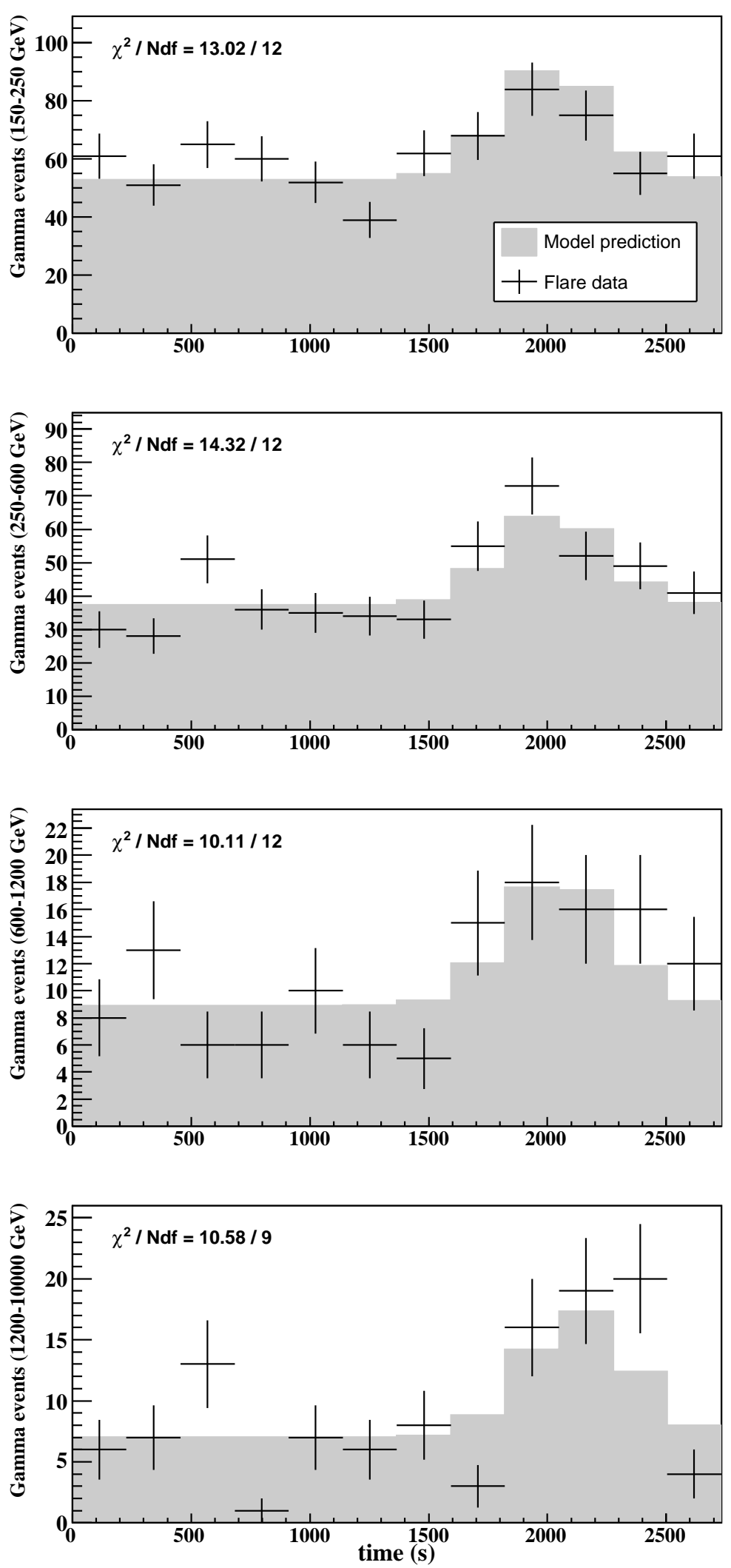

Fig. 4. Comparison of the binned MAGIC data and Likelihood function distributions to check the goodness-of-fit. The overall $\chi^{2} / \mathrm{Ndf}$ is $48.0 / 41$. 


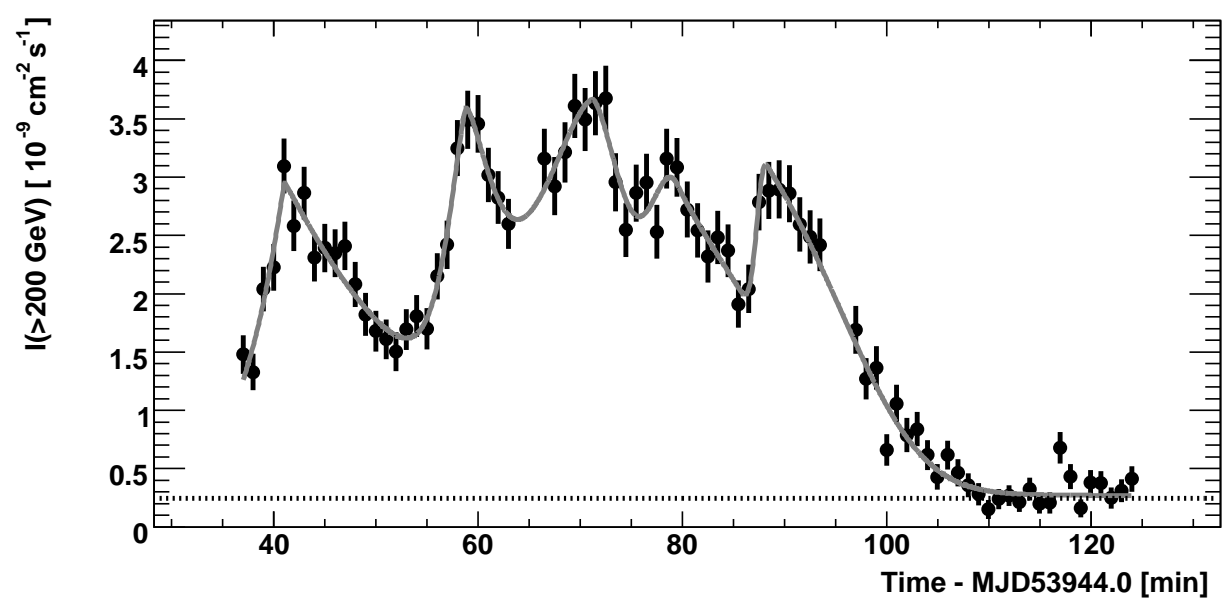

Fig. 5. The lightcurve observed from the July 26th 2006 flare of PKS2155-304 by the H.E.S.S. collaboration (fig 1 in reference [21]). The data are binned in 1-minute intervals. The line is the fit to these data of the superposition of five bursts described in the text.

\begin{tabular}{ccccc}
\hline \hline $\begin{array}{c}t_{\max } \\
{[\min ]}\end{array}$ & $A$ & $\begin{array}{c}\tau_{\mathrm{r}} \\
{\left[10^{-9} \mathrm{~cm}^{-2} \mathrm{~s}^{-1}\right]}\end{array}$ & $\begin{array}{c}\tau_{\mathrm{d}} \\
{[\mathrm{s}]}\end{array}$ & $\kappa \mathrm{s}]$ \\
\hline & & & & \\
41.0 & $2.7 \pm 0.2$ & $173 \pm 28$ & $610 \pm 129$ & $1.07 \pm 0.20$ \\
58.8 & $2.1 \pm 0.9$ & $116 \pm 53$ & $178 \pm 146$ & $1.43 \pm 0.83$ \\
71.3 & $3.1 \pm 0.3$ & $404 \pm 219$ & $269 \pm 158$ & $1.59 \pm 0.42$ \\
79.5 & $2.0 \pm 0.8$ & $178 \pm 55$ & $657 \pm 268$ & $2.01 \pm 0.87$ \\
88.3 & $1.5 \pm 0.5$ & $67 \pm 44$ & $620 \pm 75$ & $2.44 \pm 0.41$ \\
\hline
\end{tabular}

Table 2

The results of the best $\chi^{2}$ fit of the superposition of five bursts and a constant to the data shown in Figure 5. The constant term is $0.27 \pm 0.03 \times 10^{-9} \mathrm{~cm}^{-2} \mathrm{~s}^{-1}(1.1$ $\left.\mathrm{I}_{\mathrm{Crab}}\right)$. Taken from [21].

overlapping flares are collected in Tab. 2,

In addition, such a huge statistics allowed a precise analysis of the observed differential energy spectrum which, as can be seen in Fig. 6 turns out to be well described between about $200 \mathrm{GeV}$ and $5 \mathrm{TeV}$ by a broken power law such as:

$$
\begin{aligned}
& E<E_{B}: \frac{d N}{d E}=I_{0}\left(\frac{E}{1 T e V}\right)^{-\Gamma_{1}} \\
& E>E_{B}: \frac{d N}{d E}=I_{0}\left(\frac{E}{1 T e V}\right)^{\Gamma_{2}-\Gamma_{1}}\left(\frac{E}{1 T e V}\right)^{-\Gamma_{2}}
\end{aligned}
$$




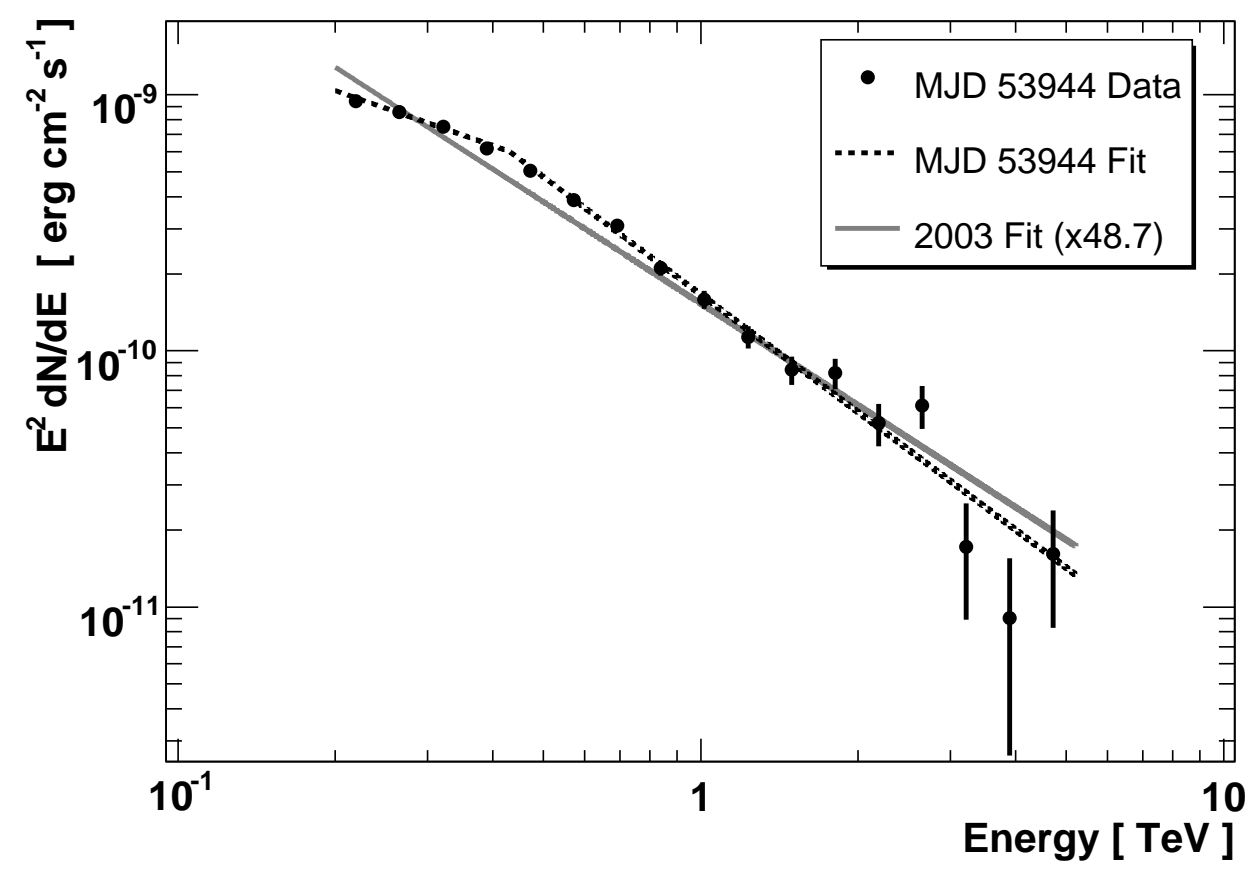

Fig. 6. The time-averaged differential energy spectrum observed from the July 26th 2006 flare of PKS 2155-304 by the H.E.S.S. collaboration (fig 3 in reference [21]). The dashed line is the best fit of a broken power-law to the data as described in the text.

where $I_{0}=(2.06 \pm 0.16 \pm 0.41) \times 10^{-10} \mathrm{~cm}^{-2} s^{-1} \mathrm{TeV}^{-1}, E_{B}=(430 \pm 22 \pm 80)$ $\mathrm{GeV}, \Gamma_{1}=2.71 \pm 0.06 \pm 0.10$ and $\Gamma_{2}=3.53 \pm 0.05 \pm 0.10$ where for each parameter the two uncertainties are the statistical and the systematic values respectively. This energy spectrum has not been corrected for the absorption of VHE gammas on the Extragalactic Background Light but corresponds to the observed one, which is precisely the input needed for the correlation study.

We wanted to test whether our method could be applied to such a kind of complex flare to eventually obtain results on any possible energy-dependent propagation delay effect. Nevertheless, since the method presented here relies on the use of the individual photon information and we have no access the actual H.E.S.S. data, Monte Carlo generated samples of simulated gammaray data following the H.E.S.S. published lightcurve and differential energy spectrum have been produced to try to understand the actual sensitivity our method would provide in spotting any non-trivial energy-time correlation, would it be present in the real data.

For that, Monte Carlo simulated gamma-ray datasets with the same amount of gammas observed by H.E.S.S., their integral lightcurve, their differential energy spectrum and their claimed energy resolution were produced. In each dataset a different value for $M_{Q G n}$ was assumed: from no energy-time correlation $\left(\hat{M}_{P} / M_{Q G n}=0\right)$ to the same value found in the fits to the MAGIC data described before, and in the linear and in the quadratic scenarios. Those 
datasets were fitted to a Likelihood function built following equation 11 in which the functions $\Gamma\left(E_{s}\right), G\left(E-E_{s}, \sigma_{E}\left(E_{s}\right)\right)$ and $F_{s}\left(t-D\left(E_{s}, M_{Q G n}, z\right)\right)$ are the same ones used for generating the simulated datasets, namely, the ones describing the H.E.S.S. data as discussed above.

Fig. 7 shows the comparison between the binned Monte Carlo dataset and the Likelihood function for the case in which a QG scale equal to the one observed in the MAGIC data is assumed. In all tested cases, the fits do recover within one sigma the $M_{Q G n}$ value used in the simulations, and this result demonstrates the reliability of our approach to analyze complex flare structures.

Moreover, our fits predict that the H.E.S.S. data could provide a sensitivity in the determination of any measurement of $M_{Q G n}$ in the linear and in the quadratic assumptions, over a factor 6 better than the one obtained with the Markarian 501 flare observed by MAGIC. By playing with our fits, we've been able to trace back the origin of this improvement to three factors:

- A factor about three comes from the fact that the redshift is about three times larger (0.116 compared with 0.034).

- Another factor about two comes from the fact that the gamma statistics is almost ten times larger (about 12 thousand gammas compared with 1.4 thousand gammas). That would produce a factor three smaller statistical uncertainty but, on the other hand, the observed MAGIC spectrum is harder (more photons at higher energies) than the H.E.S.S. one. In fact, it has been checked by playing with the spectral indices that the photons with the highest energies (above say $1 \mathrm{TeV}$ ) do play a crucial role in improving the sensitivity.

- Finally an additional small gain comes from the rich flare structure although in general, the sensitivity is dominated by the fastest risetime or falltime in the whole lightcurve.

We expect the H.E.S.S. collaboration to release soon the results of their analysis of their flare and confirm or correct our expectations.

\section{Conclusions}

In this work we've presented a new approach for the analysis of correlations between the arrival time and energy of photons that can be applied to any observed set of photons coming from astrophysical sources to search for any kind of intrinsic or propagation energy-dependent delay effects.

This approach uses directly the energy and arrival time information of each recorded photon and therefore does not require binning. Unlike other unbinned 

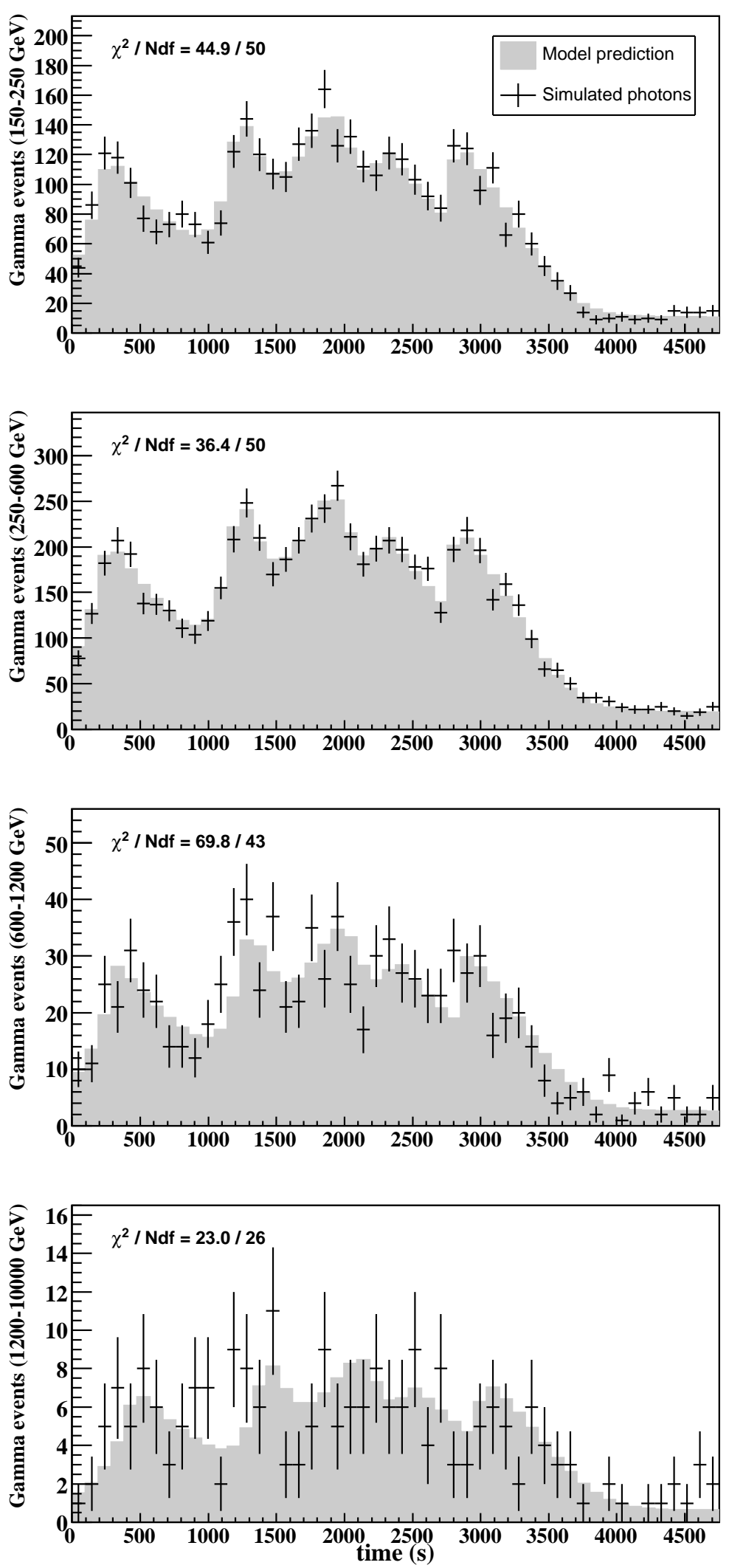

Fig. 7. Comparison of the binned HESS Monte Carlo data and Likelihood function distributions to check the goodness-of-fit. The energy intervals chosen are the same ones used in fig. 4 whereas the time bins here are of 95 seconds duration. The overall $\chi^{2} / \mathrm{Ndf}$ is $174.1 / 168$. 
approaches, the one presented should be able to study the energy versus time correlations regardless on the complexity of the flare structure and even with quite low photons statistics. The method proposed is based upon a Likelihood estimator and therefore should be statistically optimal to find the best estimator for that correlation.

This analysis technique was already applied to the Markarian 501 flare observed by the MAGIC telescope in July 2005 [20,29] and some details about the actual implementation in that case, as well as of several tests performed to verify the reliability, robustness and stability of the results have been given. For that case, the goodness-of-fit has also been discussed.

Finally, the application to a more complex practical case such as the huge flare of PKS 2155-304 observed in July 2006 by the H.E.S.S. collaboration has been discussed. Monte Carlo simulations show that the method could be reliably used to analyze that data.

\section{Acknowledgements}

We're indebted to Robert Wagner, Adrian Biland and Rudy Bock from the MAGIC Collaboration, and John Ellis, Nikolaos Mavromatos, Dimitri Nanopoulos, Alexandre Sakharov and Edward Sarkisyan-Grinbaum for many discussions and exchange of ideas during the development of this method and during its application to the MAGIC data. We're specially grateful to Adrian Biland for providing the Monte Carlo samples for some of the test of the application of this method to the MAGIC data and also for some clever suggestions.

We want to thank David Paneque for providing us with all the details of his analysis of the Markarian 501 flare and for being so obstinate in claiming that there was some funny effect there.

We also want to thank the MAGIC-IFAE group for many discussions in the early stages of the development of this method and specially Javier Rico for his critical analysis about the interpretation of its results. Moreover, we thank Rolf Bühler for suggesting a modification in equation 1 to make it more general.

Finally we want to thank all our colleagues of the MAGIC Collaboration for making possible to produce such high quality data as the one used for the application of our method to the MAGIC data. 


\section{References}

[1] Cheng, K.S., Ho, C. and Ruderman, M., ApJ 300 (1986) 500.

[2] Daugherty, J.K. and Harding, A.K., ApJ 458 (1996) 278.

[3] Ghisellini, G. and Tavecchio, MNRAS 386 (2008) 28.

[4] Bednarek, W. and Wagner, R.M., Astron. Astrophys. 486 (2008) 679.

[5] Rovelli, C. and Smolin, L., Nucl. Phys. B 331 (1990) 80.

[6] Ellis, J.R., Mavromatos, N.E. and Nanopoulos, D.V., Phys. Lett. B 293 (1992) 37.

[7] Kostelecky, V.A. and Potting, R., Phys. Lett. B. 381 (1996) 89.

[8] Amelino-Camelia, G., Ellis, J.R., Mavromatos, N.E. and Nanopoulos, D.V., Int J. Mod. Phys. A 12 (1997) 607.

[9] Gambini, R. and Pullin, J., Phys. Rev. D 59 (1999) 124021.

[10] Klinkhamer F.R., AIP Conf. Proc. 977 (2008) 181.

[11] Rovelli, C., Living Reviews in Relativity 1 (1998) 1.

[12] Ellis, J.R., Mavromatos, N.E. and Nanopoulos, D.V., "Probing models of quantum space-time foam," arXiv:gr-qc/9909085.

[13] Sarkar, S., Mod. Phys. Lett. A 17 (2002) 1025.

[14] Piran, T., Lect. Notes Phys. 669 (2005) 5.

[15] Amelino-Camelia, G., Ellis, J.R., Mavromatos, N.E., Nanopoulos, D.V. and Sarkar, S., Nature 393 (1998) 763.

[16] Biller, S.D. et al., Phys. Rev. Lett. 89 (1999) 2108.

[17] Kaaret, P., Astron. Astrophys. 345 (1999) L32.

[18] Ellis, J.R. et al., Astropart. Phys. 25 (2006) 402.

[19] Blanch, O., López, J. and Martínez, M., Astropart. Phys. 19 (2003) 245.

[20] Albert, J. et al., ApJ 669 (2007) 862.

[21] Aharonian, F. et al., ApJ 664 (2007) L71.

[22] Li, T.P. et al., J. Astron. Astrophy. 4 (2004) 583.

[23] Edelson, R.A. and Krolik, J.H., ApJ 333 (1988) 646.

[24] Mallat, S., "A Wavelet Tour of Signal Processing", Academic Press, San Diego, 1999.

[25] Ellis, J.R. et al., Astron. Astrophys. 402 (2003) 409. 
[26] Lamon, R., Produit, N. and Steiner, F., General Relativity and Gravitation 40 (2008) 1731.

[27] Bolmont, J. et al., ApJ 676 (2008) 532.

[28] Aharonian, F. et al. Phys. Rev. Lett. 101 (2008) 170402.

[29] Albert, J. et al., Phys. Lett. B 668 (2008) 253.

[30] Norris, J.P. et al., ApJ 662 (1996) 393. 